\title{
In vitro Bioefficacy of Endophytic Isolates against Citrus Nematode, Tylenchulus semipenetrans
}

\author{
K. Suganthi ${ }^{1}$, P. Vetrivelkalai ${ }^{2} *$ K. Poornima $^{1}$ and R.M. Vijayakumar $^{2}$ \\ ${ }^{1}$ Department of Nematology, ${ }^{2}$ Department of Fruit Crops, Tamil Nadu Agricultural \\ University, Coimbatore - 641003, Tamil Nadu, India \\ *Corresponding author
}

\section{A B S T R A C T}

\section{Ke y w ords \\ Endophytes, Tylenchulus semipenetrans, Egg hatching, Juvenile mortality. \\ Article Info \\ Accepted: \\ 10 April 2019 \\ Available Online: \\ 10 May 2019}

The citrus nematode, Tylenchulus semipenetrans is known to occur in all citrus growing regions. The nematode feeds upon cortical cells and results in thicker roots than normal. The nematode caused yellowing of leaves and early fruit drop. A random survey was conducted in major citrus growing regions of Tamil Nadu to isolate the endophytes from the roots of acid lime (Citrus auruntifolium) and test the efficacy against citrus nematode, T.semipenetrans. Eight bacterial and four fungal endophytes were isolated from fifty one samples and an in vitro study was conducted to test the biocontrol efficacy against citrus nematode. The results revealed that four isolates viz., VSEB3, VREB8, PMEB1 and VREF3 significantly reduced the egg hatching $(97.17,96.60,89.24$ and 88.10 percent respectively) and showed highest juvenile mortality (100, 100, 99.33 and 87 percent respectively) at $100 \%$ concentration on five days and $72 \mathrm{~h}$ of exposure respectively.

\section{Introduction}

Citrus (Citrus spp.) is third most important essential trade crop next to mango and banana in the world. Most of the fruits of this group are native to tropical and subtropical regions of South East Asia. India is the largest producer of acid lime in the world. This commercially important crop is affected by various pests, diseases and nematodes which adversely affected the fruit yield.

Tylenchulus semipenetrans, a semi endoparasitic nematode specific to all the citrus species and feed upto deeper cortical cells and sloughed the outer epidermis and cortex from the stellar region. Due to feeding, trees slowly loss vigour and productivity. It subsequently leads to slow decline also which is a responsible factor for dieback in citrus (Alb Elgawad et al., 2010). The annual yield loss in citrus due to T. semipenetrans is 30-50 percent (Baines et al., 1962). It is essential to take necessary steps to avoid the yield loss in citrus.

The present management practices are not effective in managing $T$. semipenetrans. Since 
the crop is perennial, cultural and physical methods are not practicable. The chemicals used for nematode management also banned because of its detrimental effect to natural ecosystem. So, an effective and ecofriendly nature method is to be practiced for sustainable nematode management and also conserve soil health. For that, the use of endophytes is indispensable. Endophytes are the plant associated microorganisms that form association with their host plants by colonizing the internal tissues without showing any symptoms and make them a valuable tool for improving the crop performance (Azevedo et al., 2000). Based on its ecological significance, endophytes are highly competitive to pathogens occupying the same ecological niches as pathogen and improve the interactions and defence systems of their host plants (Zhang et al., 2009). Hence, the present investigation is aimed to use the natural endophyte that resides within the plant itself to manage the nematode.

\section{Materials and Methods}

The experiment was conducted in Department of Nematology, TNAU, Coimbatore. A field survey was conducted to collect the healthy acid lime roots for the isolation of endophytes from different citrus growing regions of Tamil Nadu. The young feeder roots were collected at 30 to $60 \mathrm{~cm}$ depth and collected samples were packed in properly labelled polythene bags. The collected samples were brought to laboratory for endophytes isolation.

\section{Isolation of endophytes}

Root samples collected were washed under running tap water for free of soil. The roots are cut into small sections of $1-2 \mathrm{~cm}$ using sterile scalpel. The root sections were surface sterilized by sequential washing with $70 \%$ ethanol for 30 seconds and $2 \%$ sodium hypochlorite $(\mathrm{NaOCl})$ for 30 seconds, again with $70 \%$ ethanol for 10 seconds and finally rinsed five times in sterile distilled water (Trivedi et al., 2011). An aliquot of $0.1 \mathrm{ml}$ from final wash was transferred to plain media serves as sterile check and discarded the plates if any growth was detected in check.

The surface sterilised roots sections were cut longitudinally into two pieces and placed one in nutrient agar and other in potato dextrose agar media in such a way that the stellar region of the root touches the surface of the media and incubated in room temperature for 2-3 days for bacteria and 5-7 days for fungi. The culture grown in plates were transferred into respective plates containing fresh media and maintained as pure culture (Araujo et al., 2001).

\section{Nematode culture}

The nematode infested roots were collected from acid lime orchard at Sathya Nagar of Theni district and maintained as a monoculture in acid lime and sweet orange seedlings grown in autoclaved pot mixture under glasshouse conditions. The infested roots were gently washed in water and collected the egg masses by observing through stereozoom microscope and allowed for hatching in normal tap water. The freshly hatched out juveniles were used for inoculation at the rate of two infective juvenile per gram of soil (stage $\mathrm{J}_{2}$ ).

\section{In vitro screening of endophytic isolates}

Endophytic isolates were grown on respective broths and allowed for incubation. The culture filtrate was obtained by centrifuging the broth at $10000 \mathrm{rpm}$ for $15 \mathrm{~min}$ and passed through $0.2 \mu \mathrm{m}$ bacterial filter to avoid the cells and spores. The culture filtrates of endophytes were used for an in vitro experiment at two 
different concentrations of 50 and $100 \%$. The culture filtrate of $2 \mathrm{ml}$ was tested against two eggmasses which approximately contains 120 to 140 eggs placed in a $6 \mathrm{~cm}$ small Petridish under room temperature $\left(28-32^{\circ} \mathrm{C}\right)$. Egg masses placed in autoclaved broth and sterile distilled water serves as control. Observations were made on three days after inoculation with 24 hours interval and three replications were maintained for each isolate and the experiment was arranged in a completely randomized design. Likewise, the culture filtrate of $1 \mathrm{ml}$ was tested against 100 infective juveniles placed in a $6 \mathrm{~cm}$ small Petri dish under room temperature $\left(28-32^{\circ} \mathrm{C}\right)$. Juveniles placed in autoclaved broth and sterile distilled water serves as control. Observations were recorded on 24 hours interval and replicated thrice for each isolate and the experiment was maintained in a completely randomized design.

\section{Results and Discussion}

\section{Isolation of endophytes}

Among five districts of Tamil Nadu, two isolates from Perambalur, two from Trichy, three from Virudhunagar and five from Tirunelveli were obtained and used for in vitro assessment against citrus nematode, T.semipenetrans.

\section{Effect of endophytic isolates on $T$.} semipenetranseggs

From the observation recorded on ovicidal effect of endophytic isolates against citrus nematode eggmasses, the lowest number of eggs hatched from eggmass inoculated with isolate VSEB3(3.33) followed by VREB8 (4.00), VREF3 (12.66) and PMEB1 (13.33) at 100 percent concentration of culture filtrate after 5 days of exposure period (Table 1 and 2). All the endophytic isolates were significantly differed with each other. The results revealed that the egg hatching was inhibited by increasing the concentration and exposure time. The maximum numbers of eggs (117.66) were hatched in control followed by autoclaved broth (98.00) at 100 percent concentration on fifth day after exposure. The inhibition of egg hatching was due to the endophytes isolated from citrus may produce chitinase that breakdown the cell wall of nematode eggs (Trivedi, 2011). Similar findings by Vetrivelkalai and Sivakumar (2019) where the culture filtrates of bacterial endophytic isolates significantly reduced the egg hatching of Meloidogyne incognita and increased in exposure time and concentration which increased the inhibition of egg hatching. These findings were also in line with Jonathan and Umamaheswari (2006) and Vetrivel kalai et al., (2010).

\section{Effect of endophytic isolates on $T$. semipenetrans juveniles}

The culture filtrate of eight bacterial and four fungal isolates were tested at 50 and 100 percent concentration against $T$. semipenetrans juveniles with observations made on $24 \mathrm{~h}$ interval. The results revealed that highest juvenile mortality was observed in isolate VSEB3 (100) and VREB8 (100) at 100 percent concentration after $72 \mathrm{~h}$ of exposure to culture filtrate followed by VREF3 (99.33) and PMEB1 (87.00). Similarly, lowest juvenile mortality was observed in autoclaved broth (1.33) and no juvenile mortality was observed in control (sterile distilled water) (Table 3 and 4). Treatment of tomato plants with endophytic bacteria Bacillus cereus BCM2 significantly reduced the root knot nematode infestation. Analysis of root exudates revealed that the concentration of 2,4 ditertbutylphenol, 3,3dimethyloctane and n-tridecane get increased in $B$. cereus treated plants and induced repellent effect towards towards $M$. Incognita (Li et al., 2019). 
Table.1 Effect of cell free culture filtrate of endophytic bacterial isolates citrus nematode eggs

\begin{tabular}{|c|c|c|c|c|c|c|c|}
\hline \multirow{2}{*}{ S.No. } & \multirow{2}{*}{ Isolate } & \multicolumn{5}{|c|}{ Number of eggs hatched after an exposure* } \\
\cline { 3 - 8 } & & \multicolumn{5}{|c|}{$\mathbf{5 0 \%}$} & \multicolumn{3}{c|}{ 100\% } \\
\cline { 3 - 8 } & & 3DAI & 4DAI & 5DAI & 3DAI & 4DAI & 5DAI \\
\hline \multirow{2}{*}{ PMEB1 } & 15.33 & 16.00 & 22.00 & 7.33 & 12.00 & 13.33 \\
& & $(3.91)^{\mathrm{b}}$ & $(4.00)^{\mathrm{b}}$ & $(4.69)^{\mathrm{b}}$ & $(2.70)^{\mathrm{b}}$ & $(3.46)^{\mathrm{b}}$ & $(3.65)^{\mathrm{b}}$ \\
\hline $\mathbf{2}$ & TMEB2 & 59.00 & 62.66 & 65.00 & 24.00 & 31.00 & 35.00 \\
& & $(7.68)^{\mathrm{g}}$ & $(7.91)^{\mathrm{g}}$ & $(8.06)^{\mathrm{f}}$ & $(4.89)^{\mathrm{e}}$ & $(5.56)^{\mathrm{e}}$ & $(5.91)^{\mathrm{d}}$ \\
\hline $\mathbf{3}$ & VSEB3 & 8.33 & 13.33 & 18.00 & 1.33 & 2.00 & 3.33 \\
& & $(2.88)^{\mathrm{a}}$ & $(3.65)^{\mathrm{a}}$ & $(4.24)^{\mathrm{a}}$ & $(1.15)^{\mathrm{a}}$ & $(1.41)^{\mathrm{a}}$ & $(1.82)^{\mathrm{a}}$ \\
\hline $\mathbf{4}$ & TSEB4 & 51.00 & 56.00 & 61.00 & 18.00 & 22.00 & 28.00 \\
& & $(7.14)^{\mathrm{f}}$ & $(7.48)^{\mathrm{f}}$ & $(7.81)^{\mathrm{e}}$ & $(4.24)^{\mathrm{d}}$ & $(4.69)^{\mathrm{d}}$ & $(5.29)^{\mathrm{c}}$ \\
\hline $\mathbf{5}$ & TVEB5 & 36.00 & 43.66 & 45.00 & 6.00 & 12.00 & 14.00 \\
\hline & & $(6.00)^{\mathrm{d}}$ & $(6.60)^{\mathrm{d}}$ & $(6.70)^{\mathrm{d}}$ & $(2.44)^{\mathrm{b}}$ & $(3.46)^{\mathrm{b}}$ & $(3.74)^{\mathrm{b}}$ \\
\hline $\mathbf{6}$ & TMEB6 & 43.00 & 49.66 & 53.33 & 12.66 & 17.00 & 20.00 \\
& & $(6.55)^{\mathrm{e}}$ & $(7.04)^{\mathrm{e}}$ & $(7.30)^{\mathrm{d}}$ & $(3.55)^{\mathrm{c}}$ & $(4.12)^{\mathrm{c}}$ & $(4.47)^{\mathrm{b}}$ \\
\hline $\mathbf{7}$ & TPEB7 & 64.66 & 71.33 & 75.66 & 28.66 & 33.00 & 43.00 \\
& & $(8.04)^{\mathrm{h}}$ & $(8.44)^{\mathrm{h}}$ & $(8.69)^{\mathrm{gt}}$ & $(5.35)^{\mathrm{f}}$ & $(5.74)^{\mathrm{f}}$ & $(6.55)^{\mathrm{e}}$ \\
\hline $\mathbf{8}$ & VREB8 & 29.00 & 33.00 & 36.00 & 1.33 & 3.00 & 4.00 \\
& & $(5.38)^{\mathrm{c}}$ & $(5.74)^{\mathrm{c}}$ & $(6.00)^{\mathrm{c}}$ & $(1.15)^{\mathrm{a}}$ & $(1.73)^{\mathrm{a}}$ & $(2.00)^{\mathrm{a}}$ \\
\hline $\mathbf{9}$ & Autoclaved broth & 87.00 & 97.66 & 101.33 & 80.00 & 89.33 & 98.00 \\
& & $(9.33)^{\mathrm{i}}$ & $(9.88)^{\mathrm{i}}$ & $(10.07)^{\mathrm{h}}$ & $(8.94)^{\mathrm{g}}$ & $(9.45)^{\mathrm{g}}$ & $(9.89)^{\mathrm{f}}$ \\
\hline $\mathbf{1 0}$ & Sterile distilled & 94.00 & 110.00 & 117.66 & 94.00 & 110.00 & 117.66 \\
& water & $(9.69)^{\mathrm{j}}$ & $(10.48)^{\mathrm{j}}$ & $(10.84)^{\mathrm{i}}$ & $(9.69)^{\mathrm{h}}$ & $(10.48)^{\mathrm{h}}$ & $(10.84)^{\mathrm{g}}$ \\
\hline & SEd & 0.17 & 0.08 & 0.08 & 0.15 & 0.23 & 0.23 \\
\hline & CD (P=0.01) & 0.32 & 0.23 & 0.23 & 0.42 & 0.64 & 0.65 \\
\hline
\end{tabular}

*values are mean of three replications, figures in parentheses are square root transformed value

In column means followed by a different letter are significantly different from each other at 1 percent level by LSD

Table.2 Effect of cell free culture filtrate of endophytic fungal isolate on citrus nematode eggs

\begin{tabular}{|c|c|c|c|c|c|c|c|}
\hline \multirow{2}{*}{ S.No. } & \multirow{2}{*}{ Isolate } & \multicolumn{5}{|c|}{ Number of eggs hatched after an exposure* } \\
\cline { 3 - 8 } & & \multicolumn{5}{|c|}{$\mathbf{5 0 \%}$} \\
\cline { 3 - 8 } & & 3DAI & 4DAI & 5DAI & 3DAI & 4DAI & 5DAI \\
\hline \multirow{2}{*}{$\mathbf{1}$} & PVEF1 & 72.00 & 82.00 & 84.33 & 35.00 & 42.00 & 51.66 \\
& & $(8.48)^{\mathrm{d}}$ & $(9.05)^{\mathrm{d}}$ & $(9.18)^{\mathrm{d}}$ & $(5.91)^{\mathrm{d}}$ & $(6.48)^{\mathrm{d}}$ & $(7.18)^{\mathrm{d}}$ \\
\hline $\mathbf{2}$ & TNEF2 & 43.66 & 49.66 & 53.33 & 13.00 & 17.00 & 20.00 \\
& & $(6.60)^{\mathrm{b}}$ & $(7.04)^{\mathrm{b}}$ & $(7.30)^{\mathrm{b}}$ & $(3.60)^{\mathrm{b}}$ & $(4.12)^{\mathrm{b}}$ & $(4.47)^{\mathrm{b}}$ \\
\hline $\mathbf{3}$ & VREF3 & 15.33 & 16.00 & 22.00 & 7.33 & 12.00 & 12.66 \\
& & $(3.91)^{\mathrm{a}}$ & $(4.00)^{\mathrm{a}}$ & $(4.69)^{\mathrm{a}}$ & $(2.70)^{\mathrm{a}}$ & $(3.46)^{\mathrm{a}}$ & $(3.55)^{\mathrm{a}}$ \\
\hline \multirow{2}{*}{$\mathbf{4}$} & TSEF4 & 68.00 & 75.33 & 80.66 & 31.00 & 37.33 & 43.66 \\
& & $(8.24)^{\mathrm{c}}$ & $(8.67)^{\mathrm{c}}$ & $(8.98)^{\mathrm{c}}$ & $(5.56)^{\mathrm{c}}$ & $(6.11)^{\mathrm{c}}$ & $(6.60)^{\mathrm{c}}$ \\
\hline $\mathbf{5}$ & Autoclaved broth & 77.00 & 87.66 & 91.33 & 40.00 & 49.33 & 58.00 \\
& & $(8.77)^{\mathrm{e}}$ & $(9.36)^{\mathrm{e}}$ & $(9.55)^{\mathrm{e}}$ & $(6.32)^{\mathrm{d}}$ & $(7.02)^{\mathrm{d}}$ & $(7.61)^{\mathrm{d}}$ \\
\hline $\mathbf{6}$ & Sterile & 94.00 & 110.00 & 117.66 & 94.00 & 110.00 & 117.66 \\
& distilledwater & $(9.69)^{\mathrm{f}}$ & $(10.48)^{\mathrm{f}}$ & $(10.84)^{\mathrm{f}}$ & $(9.69)^{\mathrm{e}}$ & $(10.48)^{\mathrm{e}}$ & $(10.84)^{\mathrm{e}}$ \\
\hline & SEd & 0.10 & 0.06 & 0.07 & 0.11 & 0.04 & 0.18 \\
\hline & CD (P=0.01) & 0.32 & 0.18 & 0.22 & 0.35 & 0.11 & 0.55 \\
\hline
\end{tabular}

*values are mean of three replications, figures in parentheses are square root transformed value

In column means followed by a different letter are significantly different from each other at 1 percent level by LSD 
Table.3 Effect of cell free culture filtrate of bacterial isolates on citrus nematode juveniles

\begin{tabular}{|c|c|c|c|c|c|c|c|}
\hline \multirow{3}{*}{ S.No. } & \multirow{3}{*}{ Isolate } & \multicolumn{6}{|c|}{ Number of dead juveniles after an exposure* } \\
\hline & & \multicolumn{3}{|c|}{$\mathbf{5 0 \%}$} & \multicolumn{3}{|c|}{$100 \%$} \\
\hline & & $24 \mathrm{~h}$ & $48 h$ & $72 \mathrm{~h}$ & $24 \mathrm{~h}$ & $48 h$ & $72 \mathrm{~h}$ \\
\hline 1 & PMEB1 & $\begin{array}{l}64.66 \\
(8.04)^{c}\end{array}$ & $\begin{array}{c}71.33 \\
(8.44)^{\mathrm{c}}\end{array}$ & $\begin{array}{l}74.33 \\
(8.62)^{\mathrm{c}}\end{array}$ & $\begin{array}{l}77.66 \\
(8.81)^{b}\end{array}$ & $\begin{array}{l}84.33 \\
(9.18)^{b}\end{array}$ & $\begin{array}{l}87.00 \\
(9.32)^{b}\end{array}$ \\
\hline 2 & TMEB2 & $\begin{array}{l}34.00 \\
(5.83)^{\mathrm{d}}\end{array}$ & $\begin{array}{l}41.00 \\
(6.40)^{\mathrm{d}}\end{array}$ & $\begin{array}{l}44.00 \\
(6.63)^{\mathrm{de}}\end{array}$ & $\begin{array}{l}54.66 \\
(7.39)^{\mathrm{c}}\end{array}$ & $\begin{array}{l}61.66 \\
(7.85)^{\mathrm{cd}}\end{array}$ & $\begin{array}{l}64.66 \\
(8.04)^{d}\end{array}$ \\
\hline 3 & VSEB3 & $\begin{array}{c}87.33 \\
(9.34)^{\mathrm{a}}\end{array}$ & $\begin{array}{c}96.33 \\
(9.81)^{\mathrm{a}}\end{array}$ & $\begin{array}{c}100.00 \\
(10)^{\mathrm{a}}\end{array}$ & $\begin{array}{c}97.33 \\
(9.86)^{\mathrm{a}}\end{array}$ & $\begin{array}{r}100.00 \\
(10)^{\mathrm{a}}\end{array}$ & $\begin{array}{c}100.00 \\
(10)^{\mathrm{a}}\end{array}$ \\
\hline 4 & TSEB4 & $\begin{array}{l}27.33 \\
(5.22)^{\mathrm{f}}\end{array}$ & $\begin{array}{l}34.33 \\
(5.85)^{\mathrm{f}}\end{array}$ & $\begin{array}{l}39.33 \\
(6.27)^{f}\end{array}$ & $\begin{array}{l}45.66 \\
(6.75)^{\mathrm{e}}\end{array}$ & $\begin{array}{l}52.66 \\
(7.25)^{\mathrm{f}}\end{array}$ & $\begin{array}{l}57.33 \\
(7.57)^{\mathrm{f}}\end{array}$ \\
\hline 5 & TVEB5 & $\begin{array}{c}35.33 \\
(5.94)^{d}\end{array}$ & $\begin{array}{l}42.66 \\
(6.53)^{d}\end{array}$ & $\begin{array}{l}47.66 \\
(6.90)^{d}\end{array}$ & $\begin{array}{c}57.33 \\
(7.57)^{\mathrm{c}}\end{array}$ & $\begin{array}{l}64.33 \\
(8.02)^{c}\end{array}$ & $\begin{array}{l}70.66 \\
(8.40)^{\mathrm{c}}\end{array}$ \\
\hline 6 & TMEB6 & $\begin{array}{c}33.00 \\
(5.74)^{d}\end{array}$ & $\begin{array}{l}40.33 \\
(6.35)^{\mathrm{d}}\end{array}$ & $\begin{array}{l}45.33 \\
(6.73)^{\mathrm{de}}\end{array}$ & $\begin{array}{c}50.66 \\
(7.11)^{d}\end{array}$ & $\begin{array}{l}57.66 \\
(7.59)^{\mathrm{de}}\end{array}$ & $\begin{array}{l}62.66 \\
(7.91)^{\mathrm{de}}\end{array}$ \\
\hline 7 & TPEB7 & $\begin{array}{c}30.00 \\
(5.47)^{\mathrm{e}}\end{array}$ & $\begin{array}{l}37.66 \\
(6.14)^{\mathrm{e}}\end{array}$ & $\begin{array}{l}42.66 \\
(6.52)^{\mathrm{ef}}\end{array}$ & $\begin{array}{l}47.33 \\
(6.87)^{\mathrm{de}}\end{array}$ & $\begin{array}{l}55.33 \\
(7.43)^{\mathrm{ef}}\end{array}$ & $\begin{array}{c}60.33 \\
(7.76)^{\mathrm{ef}}\end{array}$ \\
\hline 8 & VREB8 & $\begin{array}{c}74.33 \\
(8.62)^{\mathrm{b}}\end{array}$ & $\begin{array}{l}81.66 \\
(9.03)^{b}\end{array}$ & $\begin{array}{l}87.33 \\
(9.34)^{b}\end{array}$ & $\begin{array}{c}97.00 \\
(9.84)^{\mathrm{a}}\end{array}$ & $\begin{array}{c}100.00 \\
(10)^{\mathrm{a}}\end{array}$ & $\begin{array}{c}100.00 \\
(10)^{\mathrm{a}}\end{array}$ \\
\hline 9 & Autoclaved broth & $\begin{array}{c}0.00 \\
(0.71)^{\mathrm{g}}\end{array}$ & $\begin{array}{c}0.00 \\
(0.71)^{\mathrm{g}}\end{array}$ & $\begin{array}{c}0.66 \\
(1.080)^{\mathrm{g}}\end{array}$ & $\begin{array}{c}0.00 \\
(0.71)^{\mathrm{f}}\end{array}$ & $\begin{array}{c}1.00 \\
(1.224)^{\mathrm{g}}\end{array}$ & $\begin{array}{c}1.66 \\
(1.288)^{\mathrm{g}}\end{array}$ \\
\hline 10 & $\begin{array}{l}\text { Sterile distilled } \\
\text { water }\end{array}$ & $\begin{array}{c}0.00 \\
(0.71)^{\mathrm{g}}\end{array}$ & $\begin{array}{c}0.00 \\
(0.71)^{\mathrm{g}}\end{array}$ & $\begin{array}{c}0.00 \\
(0.71)^{\mathrm{h}}\end{array}$ & $\begin{array}{c}0.00 \\
(0.71)^{\mathrm{f}}\end{array}$ & $\begin{array}{c}0.00 \\
(0.71)^{\mathrm{h}}\end{array}$ & $\begin{array}{c}0.00 \\
(0.71)^{\mathrm{h}}\end{array}$ \\
\hline & SEd & 0.12 & 0.11 & 0.12 & 0.09 & 0.14 & 0.11 \\
\hline & $\mathrm{CD}(\mathrm{P}=0.01)$ & 0.36 & 0.31 & 0.36 & 0.27 & 0.39 & 0.31 \\
\hline
\end{tabular}

*values are mean of three replications, figures in parentheses are $\sqrt{n}+0.5$ transformed value

In column means followed by a different letter are significantly different from each other at 1 percent level by LSD

Table.4 Effect of cell free culture filtrate of endophytic fungal isolate on citrus nematode juveniles

\begin{tabular}{|c|c|c|c|c|c|c|c|}
\hline \multirow{3}{*}{ S.No. } & \multirow{3}{*}{ Isolate } & \multicolumn{6}{|c|}{ Number of dead juveniles after an exposure* } \\
\hline & & \multicolumn{3}{|c|}{$50 \%$} & \multicolumn{3}{|c|}{$100 \%$} \\
\hline & & 24h & $48 \mathrm{~h}$ & $72 \mathrm{~h}$ & 24h & $48 h$ & 72h \\
\hline 1 & PVEF1 & $\begin{array}{c}35.33 \\
(5.94)^{b}\end{array}$ & $\begin{array}{l}42.33 \\
(6.51)^{b}\end{array}$ & $\begin{array}{c}47.33 \\
(6.87)^{b}\end{array}$ & $\begin{array}{c}49.33 \\
(7.02)^{b}\end{array}$ & $\begin{array}{c}56.33 \\
(7.50)^{\mathrm{b}}\end{array}$ & $\begin{array}{c}61.33 \\
(7.83)^{\mathrm{b}}\end{array}$ \\
\hline 2 & TNEF2 & $\begin{array}{c}24.66 \\
(4.96)^{c}\end{array}$ & $\begin{array}{r}32.66 \\
(5.71)^{\mathrm{c}}\end{array}$ & $\begin{array}{l}36.66 \\
(6.05)^{c}\end{array}$ & $\begin{array}{c}41.66 \\
(6.45)^{\mathrm{c}}\end{array}$ & $\begin{array}{c}47.33 \\
(6.87)^{c}\end{array}$ & $\begin{array}{c}53.33 \\
(7.30)^{c}\end{array}$ \\
\hline 3 & VREF3 & $\begin{array}{c}72.66 \\
(8.52)^{a}\end{array}$ & $\begin{array}{r}78.66 \\
(8.86)^{\mathrm{a}}\end{array}$ & $\begin{array}{c}83.66 \\
(9.14)^{\mathrm{a}}\end{array}$ & $\begin{array}{c}89.33 \\
(9.45)^{\mathrm{a}}\end{array}$ & $\begin{array}{c}95.66 \\
(9.78)^{\mathrm{a}}\end{array}$ & $\begin{array}{c}99.33 \\
(9.96)^{\mathrm{a}}\end{array}$ \\
\hline 4 & TSEF4 & $\begin{array}{l}23.33 \\
(4.83)^{c}\end{array}$ & $\begin{array}{l}30.33 \\
(5.50)^{\mathrm{c}}\end{array}$ & $\begin{array}{c}35.33 \\
(5.94)^{\mathrm{c}}\end{array}$ & $\begin{array}{c}36.33 \\
(6.02)^{d}\end{array}$ & $\begin{array}{l}43.33 \\
(6.58)^{d}\end{array}$ & $\begin{array}{c}48.66 \\
(6.97)^{d}\end{array}$ \\
\hline 5 & Autoclaved broth & $\begin{array}{c}0.00 \\
(0.71)^{\mathrm{d}}\end{array}$ & $\begin{array}{c}0.00 \\
(0.71)^{\mathrm{d}}\end{array}$ & $\begin{array}{c}0.66 \\
(1.080)^{d}\end{array}$ & $\begin{array}{c}0.00 \\
(0.71)^{\mathrm{e}}\end{array}$ & $\begin{array}{c}1.00 \\
(1.224)^{\mathrm{e}}\end{array}$ & $\begin{array}{c}1.66 \\
(1.288)^{\mathrm{e}}\end{array}$ \\
\hline \multirow[t]{3}{*}{6} & $\begin{array}{l}\text { Sterile distilled } \\
\text { water }\end{array}$ & $\begin{array}{c}0.00 \\
(0.71)^{d}\end{array}$ & $\begin{array}{c}0.00 \\
(0.71)^{\mathrm{d}}\end{array}$ & $\begin{array}{c}0.00 \\
(0.71)^{\mathrm{e}}\end{array}$ & $\begin{array}{c}0.00 \\
(0.71)^{\mathrm{e}}\end{array}$ & $\begin{array}{c}0.00 \\
(0.71)^{\mathrm{f}}\end{array}$ & $\begin{array}{c}0.00 \\
(0.71)^{\mathrm{f}}\end{array}$ \\
\hline & SEd & 0.08 & 0.08 & 0.12 & 0.06 & 0.09 & 0.09 \\
\hline & $\mathrm{CD}(\mathrm{P}=0.01)$ & 0.26 & 0.24 & 0.38 & 0.21 & 0.29 & 0.28 \\
\hline
\end{tabular}

*values are mean of three replications, figures in parentheses are $\sqrt{n}+0.5$ transformed value

In column means followed by a different letter are significantly different from each other at 1 percent level by LSD 
Schouten et al., (2016) reported that the endophytes may affect nematodes either directly or by synthesizing nematicidal compounds that kills or paralyse nematodes. The three most potent nematode antagonistic compounds 4-hydroxybenzoicacid, indole-3acetic acid (IAA) and gibepyrone D were obtained from the endophytic Fusarium oxysporum 162 that plays a dual role in triggering plant resistance against nematodes and promote the plant growth. The present study results were in agreement with the above results. Hence, the promising endophytic isolates obtained from the present study may be characterized at molecular level in order to identify species level.

\section{References}

Abd-Elgawad, M., El-Mougy, N., El-Gamal, N., Abdel-Kader, M. and Mohamed, M. 2010. Protective treatments against soilborne pathogens in citrus orchards. Journal of Plant Protection Research, 50(4): 477-484.

Araujo, W.L., W. Maccheroni, Jr., C.I. Aguilar-Vildoso, P.A.V. Barroso, H.O. Saridakis and Azevedo, J.L. 2001. Variability and interactions between endophytic bacteria and fungi isolated from leaf tissues of citrus rootstocks. Can. J. Microbiol., 47: 229-236.

Azevedo, J.L., Maccheroni, J.W., Pereira, J.O., Luiz de Araujo, W. 2000. Endophytic microorganisms: a review on insect control and recent advances in tropical plants. Electron J Biotechn, 3(1): 40-65.

Baines, R.C., Martin, J.P., DeWolfe, T. A., Boswell, S.B. and Garber M.J. 1962.
Effect of high doses of D-D on soil organisms and the growth and yield of lemon trees. Phytopathology, 52:723.

Jonathan, E.I. and Umamaheswari, R. 2006. Biomanagement of nematodes infesting banana by bacterial endophytes (Bacillus subtilis). Indian Journal of Nematology, 36: 30-233.

Li, X., Hu, H., Li, J., Wang, C., Chen, S. and Yan, S. Z. 2019. Effects of the endophytic bacteria Bacillus cereus BCM2 on tomato root exudates and Meloidogyne incognita infection. Plant Disease.

Schouten, A. 2016. Mechanisms involved in nematode control by endophytic fungi. Annual reviewof phytopathology, 54, 121-142.

Trivedi, P., Spann, T. and Wang, N. 2011. Isolation and characterization of beneficial bacteria associated with citrus roots in Florida. Microbial ecology, 62(2), 324-336.

Vetrivelkalai, P., Sivakumar, M., and Jonathan, E. I. 2010. Biocontrol potential of endophytic bacteria on Meloidogyne incognita and its effect on plant growth in bhendi. Journal of Biopesticides, 3(2), 452.

Vetrivelkalai, P. and Sivakumar, M. 2019. Characterization of Antinematicidal and Antifungal Bacterial Microbes by 16s Rrna Gene Sequence. Int.J.Curr. Microbiol.App.Sci. 8(01): 25752583

Zhang, C., Yin, L., and Dai, S. 2009. Diversity of root-associated fungal endophytes in Rhododendron fortunei in subtropical forests of China. Mycorrhiza, 19(6), 417-423.

\section{How to cite this article:}

Suganthi, K., P. Vetrivelkalai, K. Poornima and Vijayakumar, R.M. 2019. In vitro Bioefficacy of Endophytic Isolates against Citrus Nematode, Tylenchulus semipenetrans. Int.J.Curr.Microbiol.App.Sci. 8(05): 1050-1055. doi: https://doi.org/10.20546/ijcmas.2019.805.123 